\title{
El nacimiento del "Emplazamiento de Producto" en el contexto de la I Guerra Mundial: Hollywood y el período 1913-1920 como marcos de referencia
}

\author{
Juan Salvador VICTORIA MAS \\ Universidad de Málaga \\ jsvictoria@uma.es \\ Alfonso MÉndiz NogUero \\ Universidad de Málaga \\ amendiz@uma.es \\ José Borja ARJONA MARTÍN \\ Centro Universitario Villanueva (UCM) \\ jbarjona@villanueva.edu
}

Recibido: 25 de mayo de 2013

Aceptado: 2 de julio de 2013

\begin{abstract}
Resumen
Las guerras mundiales han representado el comienzo de la mayor parte de las tecnologías, conceptos y formatos que tienen que ver con la comunicación actual, especialmente en lo relativo a la propaganda y la comunicación comercial. Esta investigación propone el periodo de la I Guerra Mundial como el marco de referencia más significativo en la constitución del modelo y concepto de la técnica denominada "emplazamiento de producto", aplicada a la producción cinematográfica y circunscrita, muy especialmente, a las películas de Hollywood. Para ello, determinamos un concepto basado en los distintos autores que han tratado el tema, exponemos las distintas corrientes históricas relativas a su origen, $\mathrm{y}$-finalmente- tratamos de dilucidar qué período sería aquel en el que comienzan a darse la mayor parte de los rasgos definitorios de la técnica.
\end{abstract}

Palabras clave: Emplazamiento de producto; Comunicación comercial; Películas; Hollywood; I Guerra Mundial.

\section{The birth of "product placement" in the context of the First World War: Hollywood and the period 1913-1920 as framework}

\begin{abstract}
Both the First and the Second World War meant the beginning of most of the technologies, concepts and formats that have to do with contemporary communication, especially with regard to propaganda and commercial communication. This research proposes the period of World War I as the most significant framework in establishing the model and concept of the technique named "product placement", as applied to film production and circumscribed, especially, to Hollywood movies. To do this, we determine a concept based on the different authors who have addressed the issue, we present various historical currents regarding its origin, and -finally- try to decide in what period begins to realize most of the defining features of the technique
\end{abstract}


Key words: Product placement; Commercial communication; Movies; Hollywood; First World War.

\section{Referencia normalizada}

Victoria Mas, J. S., Mendiz Noguero, A. y Arjona Martín, J. B. (2013). El nacimiento del "Emplazamiento de Producto" en el contexto de la I Guerra Mundial: Hollywood y el período 1913-1920 como marco de referencia. Historia y Comunicación Social, vol. 18, págs. 139-155.

Sumario: 1. Introducción. 2. Distintas posturas entorno a su origen. 3. El Product Placement en el Hollywood de la I Guerra Mundial. 4. Consolidación del Product Placement a partir de 1920. 5. Conclusiones. 6. Bibliografía.

\section{Introducción}

A finales de la década de los 90 se inauguró en España la investigación sobre el "emplazamiento de producto" (product placement, a partir de ahora pp). Aceptando implícitamente la noción de pp propuesta por Balasubramanian (1994), los investigadores Méndiz $(1998,2000)$ y Victoria (1999a, 1999b) desarrollaron una línea de trabajo sustentada en dos hipótesis: una, el emplazamiento es paradigma de la comunicación presente y futura por ser ejemplo de integración de medios y mensajes; y dos, su razón de ser más profunda radica en la hibridación que lleva consigo.

En este sentido, la noción de Transmodernidad (Magda, 2004) representa un nuevo macrocontexto especialmente adecuado para pensar el presente de la comunicación, y en particular, el de esta técnica publicitaria. Con el contexto transmoderno -como es sabido- se recuperan los retos pendientes de la Modernidad, asumiendo las críticas postmodernas y superándolas. A pesar de todo el creciente escepticismo, sí existe, al menos, un "gran relato" que contar: su nombre es Globalización; y, por cierto, se construye frecuentemente de forma híbrida.

La línea trazada por Méndiz y Victoria fue respaldada por el reconocido trabajo de Gupta, Balasubramanian y Klassen (2000); y, en nuestro país, fue continuada por las aportaciones de Baños y Rodríguez 2003; Del Pino 2004; Del Pino y Olivares 2006; Méndiz 2006; Victoria 2010; Martín 2010 y Cristófol 2010. La aceptación de dicho concepto puede considerarse definitiva con la reciente investigación de Walton (2010).

Con la perspectiva que nos ofrecen estos años -especialmente fecundos en la investigación sobre el pp- nos parece ahora conveniente la revisión de su nacimiento. En síntesis, nuestra hipótesis proclama que el origen del pp podría situarse en dos marcos de referencia: uno geográfico (Hollywood) y otro temporal (1913-20), ambos con la I Guerra Mundial de fondo. Pero, antes de explicar el porqué, deberemos indicar qué otras propuestas se han realizado en lo referente a su comienzo como técnica; y, previamente a ello, clarificar qué debemos entender por pp. Es decir, primero debemos estar seguros de que hablamos de lo mismo, porque si no, fácilmente asigna- 
remos orígenes distintos a una forma comunicacional que ha sido cambiante desde siempre.

A la vista de la producción científica antes señalada, y partiendo de su nota más caracterísitica (la hibridación, ya indicada por Balasubramaniam [1994]), podemos decir que actualmente se acepta una noción de pp que contempla los siguientes puntos:

1. Comunicación dentro del mix de comunicación

2. en el ámbito de las Relaciones Públicas

3. llevada a cabo principalmente por las marcas

4. gestionada por los tres agentes implicados: productora audiovisual, intermediario y marca comercial; en la que

5. el sujeto de pp recibe exposición y un conjunto de beneficios relativos a su comunicación estratégica

6. el público es ya capaz de reconocer ese emplazamiento como resultado de una acción promocional

7. y cuya contrapartida se relaciona con una colaboración diversa (monetaria, de ahorro a la producción o de ayuda a la promoción del evento), además de con una posible contribución a la narración.

Con esta definición como punto de partida, vamos a tratar de resolver la cuestión del origen histórico del product placement.

\section{Distintas posturas en torno a su origen}

Desde los orígenes del Séptimo Arte las películas han mostrado marcas comerciales en el desarrollo de la trama como consecuencia de su pretensión de realismo. El rodaje en plena calle supone que la escena mostrará coches con logotipos más o menos visibles, carteles pegados en las paredes, etc. Pero estas apariciones no son fruto de un acuerdo comercial, sino que son objetos necesarios para la escena: los denominados props; algo muy lejano - por tanto - de lo que hoy se conoce como product placement. Este término no aparece como técnica de promoción y marketing hasta los años ochenta. El primero en utilizarlo es Harmetz (1983), aunque era ya empleada en la industria de Hollywood desde tiempo atrás.

Sobre el origen del product placement han cristalizado, en los últimos treinta años, dos posturas antagónicas: la "minimalista", que sitúa el nacimiento en los años 40, y la "maximalista", que se remonta casi a los orígenes del cine. Vamos a exponer una y otra.

La postura "minimalista" goza de una amplia aceptación. Muchos investigadores han señalado que Alma en suplicio (Mildred Pierce, 1945), dirigida por Michael Curtiz, es el primer caso documentado de product placement, porque -así lo afir- 
man- la productora del filme se comprometió a mostrar el whisky Jack Daniels en diversas escenas. Spillman (1985) apuntó la hipótesis; Reed (1989) la divulgó, y Nebenzhal y Secunda (1993) inmortalizaron el dato. Con posterioridad, la mayoría de los investigadores (Baker \& Crawford 1995, Baños y Rodríguez 2003, Del Pino y Olivares 2006) reiteraron que era la primera inserción de producto a cambio de una contraprestación económica documentada. Sin embargo, basta un visionado atento de la película (disponible en VHS desde 1990 y en DVD desde 2003) para comprobar que en ninguna de las múltiples escenas en que aparecen botellas de whisky puede verse la etiqueta de Jack Daniels, ni tampoco la forma que cualquier consumidor avezado identifica con las botellas de esa marca.

Lo curioso de ese mito, que se ha mantenido hasta la fecha, es que ya con anterioridad se habían señalado algunos precedentes, pero nadie les había prestado atención: todos los autores señalaban indefectiblemente el filme de Curtiz como el primero en emplear esa técnica. Charles Eckert (1978), el primero que investigó el fenómeno desde una perspectiva académica, había señalado ya que esta técnica era frecuente en los filmes de los años treinta; y, mucho antes, el crítico P. S. Harrison (1931), editor del Harrison's Reports and Film Review, había denunciado el empleo abusivo de marcas en las películas de su época, que parecían responder a un acuerdo comercial no reconocido. A propósito de It Pays to advertise, un filme que transcurre en una agencia de publicidad, había escrito lo siguiente:

The Paramount Picture It Pays to advertise is nothing but a billboard of immense size. I have not been able to count all the nationally advertised articles that are spoken of by the characters, but some of them are the following: Boston Garters, Arrow Collars, Manhattan Shirts, Colgate Cream, Gillette Razors, B.V.D., Hart, Shaeffner \& Marx clothes, Listerine, Victor phonographs, Murad cigarrettes, Florsheim shoes... But the most subtle thing is the brand "13 Soap, unlucky for Dirt". A trademark such as this does not, of course, exist; but I understand that Paramount has made the picture for the purpose of making a trademark out of it. My information is to the effect that Colgate has offered \$250.000 for it, and that Paramount is asking \$ 500.000 (1931: 6).

Sea o no cierto ese ofrecimiento de dinero para comprar una marca inventada en la pantalla, la realidad es que todas las otras marcas sí aparecen en el filme, y que otras películas de los años treinta muestran o citan marcas reales de manera explícita. Baños y Rodríguez (2003: 51) mencionan el caso de Piloto de pruebas (1938), en el que Clark Gable y Spencer Tracy piden en un bar dos Coca-Colas bien frías; sin embargo, indican que parece tratarse de algo casual, un ejemplo precursor de los actuales emplazamientos verbales de la marca.

Frente a este origen del pp en los años 40, en fecha reciente se ha configurado una postura que hemos calificado de "maximalista". Newell, Salmon y Chang (2006: 574) han defendido que el primer ejemplo de esta técnica aparece ya en un filme de 1896: en concreto, en Lausanne, Défilé de Be Batallion, de los hermanos Lumière, donde puede verse un cartel del jabón Sunlight posicionado de forma estratégica. Esta exposición de la marca en la pantalla no fue algo casual, sino consecuencia de un acuerdo gestionado a tres bandas por los hermanos Lumière, productores del filme, 
Lever Brother (antecedente de la actual Unilever, que entonces era propietaria de jabones Sunlight) y Lavanchy Clark, productor de cine y representante de ventas de dicha compañía. En la película se observa que, mientras tiene lugar el desfile, aparece entre el público un señor con una carretilla de Jabones Sunlight que se coloca estratégicamente en primera fila, quedando destacado a la derecha del plano, y ocupando un lugar preferente ante la cámara.

A una pregunta que le formulamos por escrito, Lesley Owen-Edwards, Responsable de Archivos de Unilever en Inglaterra, nos confirmó que este emplazamiento es el resultado de un acuerdo comercial ${ }^{1}$. En su carta nos decía que la marca se siente muy orgullosa de haber sido la pionera en el empleo de esta técnica, y que reconoce a Francois Lavanchy Clark el mérito de haber intuido las oportunidades de negocio que el cine ofrecía a su empresa para llegar a sus potenciales consumidores.

Afirma Lesley que Lavanchy Clark, negociante suizo que distribuía las películas de los hermanos Lumière en Europa, era también el representante en Suiza de Jabón Sunlight, y tuvo la idea de crear sinergia entre los dos negocios, utilizando el cine para promocionar los productos.

En todo caso, conviene señalar que el emplazamiento en cuestión cumple varios de los requisitos expuestos en la definición inicial: 1) Comunicación llevada a cabo por las marcas; 2) gestionada por los tres agentes implicados: productora audiovisual (hermanos Lumière), intermediario (Lever Brother) y marca comercial (Jabones Sunlight); 3) en la que el sujeto de pp recibe exposición y un conjunto de beneficios relativos a su comunicación estratégica. Pero no cumple los otros 4 elementos de la definición: 1) Comunicación dentro del mix de comunicación; 2) en el ámbito de las Relaciones Públicas; 3) cuya contrapartida se relaciona... con una posible contribución a la narración; y, sobre todo, 4) el público es ya capaz de reconocer ese emplazamiento como resultado de una acción promocional.

Por tanto, en puridad no podemos afirmar que éste sea el primer caso de product placement de la historia del cine. Sí podemos identificarlo como el primer antecedente, fehacientemente documentado.

Otro tanto sucede con otros casos en los que consta acuerdo comercial. Como nos dijo Owen-Edwards en su mail, ese mismo año Lavanchy Clark acordó un nuevo emplazamiento del jabón Sunlight en otra película de los Lumière: Les Laveuses (más conocida por su título en inglés: Washing Day in Switzerland), donde se muestra a la mujer de Lavanchy y a otros familiares lavando con el Jabón Sunlight en su casa, junto al Lago de Ginebra, colocando las cajas de jabón en lugar destacado, delante de la acción, y ocupando así un papel protagonista (Newell, Salmon y Chang, 2006: 579). 


\section{El "Product Placement" en el Hollywood de la I Guerra Mundial}

Durante los años 1913-1920 la creciente aceptación del cine norteamericano corre paralela a fuertes cambios económicos $-\mathrm{y}$ a las correspondientes alteraciones de los modelos de consumo- que tenían lugar en los Estados Unidos y en el resto del mundo. Entre otros, podemos destacar tres factores fundamentales (Allen 1980: 488):

- Aumento espectacular de la producción cinematográfica a partir de 1910, con la introducción de la producción masiva y de las técnicas de distribución.

- Para la exportación de esta sobreproducción, necesidad de una promoción internacional -el fenómeno se repite después de la Segunda Guerra Mundialque las redes de Hollywood pudieron pronto proporcionar.

- Reducción de la jornada laboral que aumenta el tiempo libre disponible al mismo tiempo que el entretenimiento se convierte en sí mismo en una industria.

A estos factores habría que añadir dos hechos importantes: el nacimiento de la industria cinematográfica de Hollywood a partir de 1911 (ese año se establece allí el primer gran estudio: Universal Pictures, a los que pronto se suman: Fox Films, Paramount, United Artists, etc.) y el fuerte declive que experimenta la producción cinematográfica europea durante los años de guerra. Ese descenso en Europa llega a ser tan notable que los empresarios ingleses y alemanes se lamentan de esta situación y la atribuyen a un cambio en los gustos de las audiencias: los espectadores sólo demandan películas producidas en los Estados Unidos (Eckert 1978: 4).

El periodo que nos ocupa (1913-1920) fue tan crucial que, a finales de la década de los veinte, el jefe de ventas de la Paramount podía afirmar que "la más grande agencia de venta de automóviles en el exterior son las películas americanas", y el director del US Census Bureau of Economic Programs, que "el papel del espectáculo como multiplicador es probablemente tan grande, o más, que el de cualquier otra industria" (Wasko, Phillips \& Chris 1993: 288).

Además de esto, la conexión entre el cine de Hollywood y la sociedad consumista fue establecida, al menos, en otros tres niveles:

- El acceso a las salas cinematográficas era una manifestación visible de participación en una ambiente de consumo elevado (acceso, en algunas ocasiones, a un nivel de vida de otra forma inaccesible);

- Las condiciones físicas de la exhibición establecieron una relación entre la experiencia cinematográfica y el comportamiento del consumidor; sin ir más lejos, las sedes de los cines se localizaron en los distritos comerciales de las ciudades. Es llamativo que, ya en 1910, unos grandes almacenes en Los Angeles anunciaran la apertura, en su quinta planta, de unas instalaciones cinematográficas con un aforo para mil personas (Allen 1980: 482); 
- Y, por último, las condiciones del sistema cinematográfico de Hollywood fueron perfectas desde el principio para la asunción de un papel importante en la nueva fase del capitalismo histórico que la filosofía del consumismo estaba a punto de propiciar: una industria fundada por entidades como $A T \& T, R C A$, The Rockefellers' Chase National Bank, etc., todos los grandes bancos y casas inversionistas y varias de las más grandes empresas de los Estados Unidos. Y todavía más: Hollywood hizo más que cualquier otra fuerza de la cultura del capitalismo para suavizar la operación del ciclo producción-consumición, entre otras formas, mediante la conversión de los productos en "fetiches", en objetos de valor simbólico (Eckert 1978: 2-21).

Sin embargo, a poco de terminar la I Guerra Mundial, la industria tuvo que enfrentarse con una serie de problemas graves: "una crisis de audiencia, que obligó a un rápido cambio de los contenidos de los films; una drástica reconversión industrial, que trajo aparejada una mayor concentración (propiciada por los nuevos accionistas del negocio, los inversores de la Costa Este); y finalmente, una serie de escándalos que motivaron serias protestas desde varios frentes" (Torreiro 1984: 38). Entre estos últimos se encontraban diversos movimientos de defensa de la moralidad y las buenas costumbres, que fueron los propiciadores indirectos de la primera de las acciones de autocensura de la industria cinematográfica. Frente a la amenaza de injerencia gubernamental, y sobre la base del gran poder persuasor del nuevo medio, se estableció el Código Hays. Con mucha facilidad, estas influencias y concienciaciones se relacionarán también, más adelante, con el comportamiento consumidor de los espectadores.

A pesar de esta primera crisis de crecimiento, desde 1916, y de forma creciente, la publicidad había comenzado a ser proyectada en las pantallas de cine. Muy pronto las estrellas habían sido solicitadas por los anunciantes para protagonizar sus campañas publicitarias. De hecho, algunos de los grandes mitos del cine mudo, como Pearl White y Marion Davies, pudieron ser vistos en anuncios de la compañía Coca-Cola ${ }^{2}$. El cine se percibe como un medio ideal para promover el consumo; el mito y la fantasía alrededor de las ficciones y de las estrellas se presentaban como instrumentos idóneos de mediación para el marketing de los productos de los patrocinadores. De hecho, se podría decir que, desde las lujosas películas de los años 20, "el patrocinio tal y como lo entendemos actualmente tiene sus raíces en Hollywood" (Ford \& Ford 1993: 7); patrocinio que, según estos autores, ya era frecuente entonces junto a "actividades de comunicación comercial como el merchandising, el product placement y el endorsement, y otras autodenominadas técnicas auxiliares de marketing" (Idem: 8).

Estas diversas combinaciones entre entretenimiento y promociones comerciales darían lugar a lo que después se llamó the soft sell: un descubrimiento acerca de que el creciente interés del público por las estrellas y por sus comportamientos podía ser utilizado, mediante la utilización de su glamour y atractivo dramático, para la modificación de los hábitos de consumo. Allen (1980:487) cita dos ejemplos significativos en los que se entrelazan los dos ámbitos: un pronóstico para la moda de primavera 
diseñado por la Paramount, y una descripción de la forma en que las mujeres a la moda desarrollan ideas para la decoración interior copiando los escenarios de las películas.

Stephen Harvey (1986:14) data también en esos años algunos esfuerzos concretos de Hollywood para que los espectadores compren "nuevas vidas". Harvey cuenta cómo durante la época de los grandes estudios, éstos solían alquilar a las estrellas -que tenían ligadas por contrato- para que colaborasen en la promoción de marcas en las páginas de revistas como Collier's y Modern Screen.

Pues bien, en este contexto de cooperación entre las promociones comerciales de las empresas y las del cine (por tanto, dentro del mix de comunicación) es donde aparecen las primeras menciones explícitas al emplazamiento de producto. Eckert señala explícitamente que "los pequeños dramas y comedias de 1910 a 1920, especialmente los que tratan sobre los estilos de vida contemporáneos de las clases media y alta, presentaron innumerables oportunidades para los emplazamientos de productos y nombres de marcas" (1978:4). Bastaba con presenciar estas películas para experimentar un deseo hacia los bienes materiales que ofrecían.

En cuanto a la fijación de la fecha exacta del origen del emplazamiento de producto, Eckert es aún más explícito. Indica que fue una práctica común en los filmes realizados en Norteamérica durante la Primera Guerra Mundial, si bien comenzó a practicarse unos años antes (1978:14). Como prueba de ello, aporta el testimonio de un agente publicitario de Los Angeles que se jactaba de haber colocado, en uno de los escaparates que aparecen en la película Age of Consent (1913), algunas de las marcas para las que trabajaba su agencia, especialmente Dr. West y Coca-Cola:.

"Some time ago I dressed the window of Toler's Drug Store on RKO's Age of Consent set, using conspicuous showcards and cutouts of Dr. West 's products and Bromo-Seltzer (two of my accounts). Considerable action of the drama took place before these windows and that was all to the good. But when the action swung inside the 'store' there were many long shots, hence the counter racks, labels, etc., which I had planted were almost illegible when the picture was projected. On this same set, I had hung a Coca-Cola electrolier well to the rear; its lettering was nearly undecipherable; yet the familiar design and trade-mark got across, since it was in full view of the audience for about one half hour of screening time" (Eckert 1978:14).

El proceso era bien simple y surgió de forma espontánea. De vez en cuando, los estudios solicitaban props a las marcas a cambio de la publicidad gratuita que el cine les podía dar; más adelante, aceptaron o solicitaron tarifas por poner en primer plano marcas o artículos reconocibles. De ahí a la institucionalización de la práctica sólo era cuestión de tiempo.

Por su parte, Allen (1980:488) habla de 1920 como la fecha de inicio de la técnica del emplazamiento de producto. En ese año se publicó un artículo en Printer's Ink en el que se indica cómo el enlace entre el cine y el consumo estaba siendo explícitamente explotado mediante un negocio con cierta entidad. El ejemplo que cita es el de una popular estrella infantil, Madge Evans, contratada por la World Film Company, 
y que llegó a ser el centro de una campaña de publicidad para la Madge Evans Hat Company de Nueva York, una empresa fabricante de sombreros que llevaba su mismo nombre. Para ese año, concluye este autor, las películas de Hollywood recibían gratuitamente props a cambio de su exposición nacional a una audiencia que crecía día a día (p. 489).

De esta forma, el pp vino a ser una manifestación más -aunque, sin duda, la más relevante- de esa relación mutuamente benéfica entre el cine y la sociedad de consumo. Y en ella se daban, definitivamente, las siete características que hemos visto señaladas en la definición de pp. No solo las que aparecen en los primeros filmes de la historia (1.- Comunicación llevada a cabo por las marcas; 2.- gestionada por los tres agentes implicados: productora, intermediario y marca comercial; 3 .- en la que el sujeto de pp recibe exposición en el filme) sino también aquellas derivadas de un "sistema de producción" que oficializa la práctica del pp (4.- Comunicación dentro del mix de comunicación; 5.- en el ámbito de las Relaciones Públicas; 6.- cuya contrapartida se relaciona con una contribución en la producción y/o la narración) y (7.-) en la que el público es ya capaz de reconocer ese emplazamiento como resultado de una acción promocional.

A modo de síntesis, podemos añadir también que, desde esta perspectiva, el nacimiento del pp se relaciona con:

1. La hibridación entre entretenimiento y promoción comercial (en un anticipo de lo que será típico en el marketing desde los años 80 hasta la actualidad: publirreportajes, infomercials, advergaming, etc.)

2. La fusión de los intereses de la industria del cine con los del consumo de masas y el capitalismo consumista

3. La promoción de unos determinados "estilos de vida", relacionados con el consumo

4. El "star system" de los grandes estudios y la creciente influencia de las estrellas del cine en la vida cotidiana de los espectadores.

Cada uno de estos puntos, por su importancia en el desarrollo del pp, merecería un análisis pormenorizado y exhaustivo que aquí no podemos realizar. Queda pendiente para futuros trabajos. Sólo queremos señalar su decisiva importancia en el nacimiento de esta técnica comercial. Añadamos también que quizás resulte anácronica la utilización de términos como merchandising o endorsement-como hace alguno de los autores citados- para la descripción de estas primeras acciones de comunicación a través del cine: se trata de una terminología difícilmente aplicable a la época que se describe.

En definitiva, la comunicación comercial que emergía por aquel entonces de la sociedad de consumo encontró en el cine norteamericano de la I Guerra Mundial un terreno abonado. Allí, desde su nacimiento, existió, más que en cualquier parte del mundo, un equilibro entre los intereses del arte y de la industria, ya que, de hecho, 
la industria era necesaria para la misma producción cinematográfica. Tanto es así que el cine de Hollywood ha sido considerado como la fusión necesaria de estos dos elementos. Más que una industria enfrentada con el arte, en la Costa Oeste de los Estados Unidos se supo concebir un auténtico arte industrial. Por tanto, frente a las posturas "minimalista" y "maximalista", nosotros optamos por una vía intermedia. Pensamos que el origen del pp no hay que buscarlo tanto en un filme concreto y aislado, cuanto en una época y en un contexto geográfico. En concreto, en los años 1913-1920 y en el contexto cultural del cine de Hollywood.

\section{Consolidación del PP a partir de 1920}

¿Y qué pasó después? ¿Cambió realmente el modo en que las empresas promocionaban sus productos a través de la pantalla? ¿Puede decirse que el período de 1913-1920 fue un punto de inflexión -verdadero nacimiento- de lo que hoy denominamos product palcement? Con los datos en la mano, podemos afirmar que sí hubo un cambio, y que -con altibajos- el modo de realizar el pp cambió sustancialmente a partir de la I Guerra Mundial.

Tras la crisis de 1929, y durante toda la década de los años 30, el estilo y las modas de Hollywood penetraron en todo el país. Tal vez basándose en esa extensión del pp, diversos autores (p. ej.: Karrh 1995) datan su nacimiento en estos años, cuando los efectos de la Depresión se hacían notar también en la industria cinematográfica. Y es que, gracias a lo acontecido en el período 1913-20, la técnica del pp empezaba a ser bastante habitual. En los años 30 se alcanza una gran sofisticación en las prácticas de "comunicación conjunta" entre Hollywood y los anunciantes, y se supera con creces la etapa de las marcas ficticias (" $A C M E$ reality", en terminología de Kerns [1993]: creación de marcas ficticias, como la archiconocida marca ACME de los dibujos animados de la Warner).

A partir de mediados de la década de los 70, la aparición de marcas en las películas experimenta, en los Estados Unidos, un crecimiento extraordinario. De hecho, numerosos autores (Harmetz 1983, Clark 1989, Troup 1991, Snyder 1992, Balasubramanian 1994 y Brett 1995) datan en estos años el nacimiento del product placement tal y como lo conocemos actualmente. Diversas circunstancias concurren durante estos años: la asunción del modelo derivado de la crisis de los grandes estudios (1948), que propicia la expansión de las pequeñas productoras (más sensibles al ahorro y a los ingresos por $\mathrm{pp}$ ); la tendencia general de Hollywood a rodar en localizaciones exteriores (lo que facilita la proliferación de marcas en el filme); la multiplicación de las audiencias cinematográficas debido a la consolidación de las nuevas tecnologías del vídeo; y por último, y quizá el factor más significativo, la aparición de los intermediarios o emplazadores profesionales.

Aún así, en los primeros años de la década de los 80 , no podemos decir todavía que la técnica del product placement haya alcanzado su madurez. Muchos grandes anunciantes cuestionan el valor de esta forma de comunicación no tradicional fundamen- 
talmente por dos cuestiones prácticas: el cine es un negocio demasiado arriesgado y el pp carece de un sistema claro para seleccionar sus actuaciones y medir la eficacia. Todavía hay demasiadas incertidumbres, y esto lleva a muchos anunciantes a una actitud de expectativa.

El año 1982 representa un hito importante en la historia particular del pp. Dos sucesos de distinta índole, durante este año, contribuyen a configurar el status actual de la práctica: el emplazamiento de la marca Reese's Pieces en la película E.T. (The Extra-Terrestrial), de Steven Spielberg, y la compra de la Columbia Pictures por parte de la compañía Coca-Cola. El primero hizo tangibles los beneficios potenciales del product placement y propició que la técnica comenzase a tener un reconocimiento como posible medio promocional. El segundo reafirmó la consideración del pp como técnica integrada dentro de una estrategia más amplia de comunicación. A partir de entonces, comenzó la implicación de los grandes estudios en lo que ya es considerado como un negocio: la 20th Century Fox, la MGM/United Artist, la Warner Brothers y, al final de la década de los 80 , el resto de los grandes estudios.

Desde entonces, parece percibirse un cambio significativo en las actitudes de las empresas y de las agencias de publicidad. Las transacciones monetarias, a cambio de la la inclusión de productos y marcas, se hacen habituales, aunque normalmente el pago no se realiza por emplazamientos aislados, sino que se trata ya de relaciones más a largo plazo. Aún así, los beneficios globales del pp comienzan a estimarse mayores que los cobros directos de los emplazamientos: otras prácticas, como las promociones conjuntas y las reducciones de presupuesto derivadas de la donación de productos, son percibidas de una forma más ventajosa por parte de la producción audiovisual. En definitiva, a lo largo de la década de los 80, el panorama descrito configura la práctica del product placement como una nueva industria en el seno de la industria cinematográfica.

Finalmente, el pp alcanza su definitiva madurez a finales de 1991, con la creación de la Entertainment Resources \& Marketing Association (ERMA). Esta asociación profesional nace con la idea de mejorar la imagen del pp mediante el establecimiento de unos criterios sólidos para su práctica legal y deontológica. ERMA representa, de esta forma, el reconocimiento profesional del product placement y su aceptación generalizada. Los profesionales descubren la buena predisposición hacia la práctica por parte del público, que no encuentra en absoluto forzada la aparición de marcas en las ficciones, ni siquiera la consiguiente realización de acuerdos para la consecución de este fin. Como consecuencia práctica, infinidad de marcas pueblan la inmensa mayoría de las producciones que pretenden recrear un mundo en el que estos signos comerciales están presentes. Es decir, desde 1920 el modelo no ha cambiado: sólo ha puesto el énfasis en alguno de los elementos de la definición: en los años 30, la extensión de la práctica junto a la capacidad del público para reconocerla; en los 70 , la profesionalización de su ejercicio, con la aparición de los intermediarios profesionales; en los 80, su inclusión en el mix de comunicación; y en los 90, su normalización ética y legal. 
Por tanto, y a modo de síntesis, podemos reconocer una trayectoria uniforme en la evolución del pp, una trayectoria que se inicia en el período 1913-20 y que llega a su culmen a principios de los años 90 . En todo este tiempo, la práctica del pp se integra en la estrategia de comunicación de las empresas, se acuerda por los tres agentes implicados en ella, y es potencialmente identificada por el público como una concreta acción comercial. Si esta evolución tiene un origen, sin duda debemos señalarlo en el ámbito geográfico de la producción hollywoodiense y en el período determinado por la I Guerra Mundial.

De forma sintética, podemos esquematizar así la presencia de los 7 rasgos que identifican el concepto de pp en las tres épocas en las que se ha propuesto su nacimiento:

\begin{tabular}{|l|c|c|c|}
\hline & Minimalista & 1913-1920 & Maximalista \\
\hline 1) Comunicación dentro del mix & & $\mathrm{X}$ & $\mathrm{X}$ \\
\hline 2) en el ámbito de las Relaciones Públicas; & & $\mathrm{X}$ & $\mathrm{X}$ \\
\hline 3) llevada a cabo por las marcas & $\mathrm{X}$ & $\mathrm{X}$ & $\mathrm{X}$ \\
\hline $\begin{array}{l}\text { 4) gestionada por los 3 agentes implicados: } \\
\text { productora, intermediario y marca }\end{array}$ & $\mathrm{X}$ & $\mathrm{X}$ & $\mathrm{X}$ \\
\hline $\begin{array}{l}\text { 5) el sujeto de pp recibe exposición y } \\
\text { beneficios en su comunicación estratégica }\end{array}$ & $\mathrm{X}$ & $\mathrm{X}$ & $\mathrm{X}$ \\
\hline $\begin{array}{l}\text { 6) el público es ya capaz de reconocer el } \\
\text { emplazamiento como acción promocional }\end{array}$ & & $\mathrm{X}$ & $\mathrm{X}$ \\
\hline $\begin{array}{l}\text { 7) y cuya contrapartida se relaciona con una } \\
\text { colaboración económica, además de con una } \\
\text { posible contribución a la narración }\end{array}$ & & & $\mathrm{Fu}$ \\
\hline
\end{tabular}

Fuente: Elaboración propia

\section{Conclusiones}

Al término de esta investigación sobre el origen del product placement, podemos afirmar que nuestras conclusiones hacen referencia a dos grandes aspectos. En primer lugar, tienen que ver con la determinación del período: los años 1913-1920, marcado por el trasfondo de la I Guerra Mundial; y en segundo lugar, con la localización geográfica: Hollywood y su estilo de vida florecientemente consumista.

En relación con el aspecto temporal, el periodo 1913-1920 comprende los años en los que el emplazamiento se practica por primera vez según el modelo vigente. No hay duda de que el pp nace como técnica durante la I Guerra Mundial, ya que es durante este período cuando alcanza la madurez conceptual, que incluye la noción transmoderna de "hibridación" (Balasubramanian, 1994): comunicación comercial 
mediante colaboración entre marca, productora e intermediarios, con la sutileza de una posible contribución a la narración.

En cuanto al segundo aspecto, Hollywood supone la consolidación del cine como arte y, a la vez, como industria, en una solución única. Allí se producen películas que generan por sí mismas actos de consumo y una ideología que la sitúa en el foco de las demás industrias. Es en Hollywood, y en el período 1913-1920, donde la cinematografía, que ya se consideraba Arte, se integra definitivamente en los principios de la Industria. La creciente cultura del ocio sitúa a la cinematografía de Hollywood en una posición privilegiada, de modo que las películas hollywoodienses no serán, únicamente, simples escaparates de productos, sino motor de una ideología del consumo, apoyada sobre los pilares de la narrativa.

Dada la coyuntura de la I Guerra Mundial, este aspecto geográfico se convierte en universal. La producción de Hollywood es la única que puede exhibirse también en Europa. La producción europea, y un posible distinto modelo que afectase también a la relación entre marcas y cine, está paralizada. Y no sólo por la guerra: los empresarios ingleses y alemanes atribuyen la preeminencia del cine americano también a un cambio en los gustos de las audiencias.

Desde 1916, y de forma creciente, la publicidad comienza a ser proyectada en las pantallas de cine. Muy pronto las estrellas son requeridas por los anunciantes para protagonizar sus campañas publicitarias. El cine se percibe como un medio ideal para promover el consumo; el mito y la fantasía alrededor de las ficciones y de las estrellas se presentan como instrumentos idóneos para la promoción de los productos. Desde las lujosas películas de los años 20 , el patrocinio tal y como lo entendemos actualmente hunde sus raíces en Hollywood, y los autores reconocen unánimemente que esta técnica de comunicación atrae consigo al resto de las técnicas que hoy llamamos "no convencionales" (entre las que estaría el pp).

En este contexto de cooperación entre las promociones comerciales de las empresas y las del cine (por tanto, dentro del mix de comunicación) es donde aparecieron las primeras menciones explícitas al emplazamiento de producto. Bastaba con presenciar estas películas para experimentar un deseo hacia los bienes materiales que ofrecían. En cuanto a la fijación de la fecha exacta del origen del emplazamiento de producto, compartimos básicamaente la tesis de Eckert acerca de que fue una práctica común, por primera vez, en los filmes realizados en Norteamérica durante la I Guerra Mundial.

De esta forma, el pp vino a ser una manifestación más -aunque, sin duda, la más relevante- de esa relación mutuamente benéfica entre el cine y la sociedad de consumo. Y en ella se daban, definitivamente, las siete características que hemos visto señaladas en la definición de pp. No solo las que aparecen en los primeros filmes de la historia (1.- Comunicación llevada a cabo por las marcas; 2.- gestionada por los tres agentes implicados: productora, intermediario y marca comercial; 3 .- en la que el sujeto de pp recibe exposición en el filme) sino también aquellas derivadas de un "sistema de producción" que oficializa la práctica del pp (4.- Comunicación 
dentro del mix de comunicación; 5.- en el ámbito de las Relaciones Públicas; 6.- cuya contrapartida se relaciona con una contribución en la producción y/o la narración) y en la que el público es ya capaz de reconocer ese emplazamiento como resultado de una acción promocional (7.-).

Como colofón, podemos añadir también que, desde esta perspectiva, el nacimiento del pp se relaciona con la hibridación entre entretenimiento y promoción comercial, con la fusión de los intereses de la industria del cine con los del consumo de masas y la promoción de unos determinados "estilos de vida", y con el "star system" de los grandes estudios y la creciente influencia de las estrellas del cine en la vida cotidiana de los espectadores.

En las décadas siguientes, el modelo no cambiará. Sólo pondrá el acento en alguno de los rasgos de la definición del pp: su cotidianidad como práctica, su profesionalización, su inclusión en el mix de comunicación o su normalización ética y legal.

\section{Bibliografía}

ALLEN, J. (1980): “The Film Viewer as Consumer", Quarterly Review of Film Studies, 1980 (Fall), 5 (IV): 481-99

BALASUBRAMANIAN, S. K. (1994): "Beyond Advertising and Publicity: Hybrid Messages and Public Policy Issues". Journal of Advertising 23, no. 4, 29-46.

BAKER, M. J. \& CRAWFORD, H. A. (1995), Product Placement, Department of Marketing, University of Strathclyde, Glasgow.

BAÑOS, M. y RODRÍGUEZ, T. (2003): Product placement. Estrella invitada: la marca. Madrid: CIE-Dossat.

BAÑOS, M. (2004). "Internet ¿Una puerta abierta a la publicidad?" Icono14, 2 (1).

BRETT, S. J. (1995): The Shrinking Screen: The Increasing Intersection of Hollywood Films and Television Programming, Ph. D. dissertation, Northwetern University.

CLARK, E. (1989): La publicidad y su poder (Las técnicas de provocación al consumo), Barcelona: Planeta.

CRISTOFOL, C. (2010): Publicidad y discurso de la moda: La hibridación entre publicidad e información en los contenidos de las revistas femeninas de alta gama: Glamour, Vogue, Marie Claire, Elle, Cosmopolitan, Telva y Woman. Tesis Doctoral en Comunicación Audiovisual y Publicidad (Universidad de Málaga).

DEL PINO, C. (2004): Marcas y ficción televisiva: El Product Placement en las Teleseries Españolas (1991-2002). Tesis Doctoral en Publicidad. Defendida el 5 de julio de 2004. Calificación: Apto Cum Laude por unanimidad.

DEL PINO, C. y OLIVARES, F. (2006): Brand Placement: integración de marcas en la ficción audiovisual, Gedisa, Barcelona.

ECKERT, Ch. (1978): “The Carole Lombard in Macy's Window”, Quarterly Review of Film Studies, 1978 (Fall), 1(III): 1-21.

FORD, B \& FORD, J. (1993): Television and Sponsorship, Oxford: Focal Press. 
GUPTA, P., BALASUBRAMANIAN, S Y KLASSEN, M. (2000), "Viewers Evaluations of Product Placement in Movies: Public Policy Issues and Managerial Implications", Journal of Current Issues and Research in Advertising, 22 (2), 2000 (Fall), 41-52.

HARMETZ, A. (1983): "Fox to sell product plugs in movies", The New York Times, 20 December 1983, p. C19.

HARRISON, P. S. (1931): “Does It Pays to Advertise?" Harrison's Reports and Film Review, 28-February-1931, p. 6.

HARVEY, S. (1986): “They Bought It At The Movies”, Adweek, 8 de septiembre de 1986, p. 14.

KARRH, JA (1995): "Brand placements in feature film: the practitioners' view", in: ed.Madden, C. S., Proceeding of the 1995 Conference of the American Academy of Advertising, Waco, TX: American Academy of Advertising, pp.182-188.

KERNS, H. D. (1993): "For Whom the Bell Tolls and the Birth of the New Auteur Movement", Annual Conference of the International Visual Literacy Association.

MARTÍN, A. (2010): Brand placement del sector del automóvil en el cine norteamericano contemporáneo. Tesis Doctoral en Comunicación Audiovisual y Publicidad (Universidad de Málaga).

MÉNDIZ, A. (1998), "El Product placement como estrategia de comunicación: orígenes y delimitación conceptual" Comunicación sin fronteras, Actas de las XII Jornadas Internacionales de Comunicación, UNAV, Texitex, Pamplona, 1998, 344-381.

--- (2000): Nuevas formas publicitarias: Patrocinio, Product Placement, Publicidad en Internet. Málaga: SPICUM.

--- (2006): "Marcas en el cine, en las series... ¡Hasta en la sopa! ¿No hay límites para el engaño en publicidad?"; en: A. Méndiz y C. Cristófol (coords.), Falsedad y Comunicación. Publicidad engañosa, Información falsa, imagen manipulada, S.P.I.C.U.M., Colección Debates nº 23, Universidad de Málaga, pp. 29-44.

NEBENZHAL, I. \& SECUNDA, E., "Consumers Attitudes Toward Product Placement in Movies", International Journal of Advertising, $\mathrm{n}^{\circ} 12,1993,1-11$.

NEWELL, J.; SALMON, Ch. T. \& CHANG, S. (2006): "The hidden history of Product Placement", Journal of Broadcasting \& Electronic Media, v. 50, issue 4, 2006: 575-594.

PENDERGRAST, M. (1993): Dios, Patria y Coca-Cola, Vergara, Buenos Aires.

PINO, C. del y OLIVARES, F. (2006): Brand Placement. Integración de marcas en la ficción audiovisual. Barcelona: Gedisa.

REED, J. D., "Plugging Away in Hollywood", Time, 103, 2-Enero-1989, p. 4.

RODRÍGUEZ, R. M. (2004): Transmodernidad, Barcelona: Anthropos.

SNYDER, S. L. (1992): "Movies and product placement: is Hollywood turning films into commercial speech?" University Illinois Law Review, 1 (1992): 301-337.

SPILLMAN, Susan, "Marketers race to leave brand on films", Advertising Age, 1 July 1985, p. 56.

TORREIRO, M. (1984): “'Dossier'. Hollywood en silencio. La censura en Hollywood", Dirigido por, 113, marzo de 1984, p. 39 
TROUP, M. L. (1991). The captive audience: A content analysis of product placements in motion pictures. Unpublished master's thesis, Florida State University.

VICTORIA, J. S. (2010): Hollywood i marki. Product placement w kinie amerykanskim. Wydawnictwo SWPS Academica, Warszawa.

--- (1999a): Presencia, Comunicación y Estrategia de las marcas comerciales en la ficción cinematográfica de Hollywood. Universidad de Málaga: SPICUM.

--- (1999b): Hollywood y las marcas (Product Placement). Madrid: Newbook.

WALTON, A. (2010): "The Evolution of Product Placement in Film". The Elon Journal of Undergraduate Research in Communications, Vol. 1, No. 1 • Winter 2010: $70-85$.

WASKO, J.; PHILLIPS, M. \& CHRIS, P. (1993): "Hollywood meets Madison Avenue: the commercialization of US Films", Media, Culture and Society, University of Oregon, 15 (2), 271-293.

\section{Notas}

1 E-mail de Lesley Owen-Edwards, fechado en UK el 09-11-2010, en contestación a nuestro escrito del 06-11-2010. Entre otras cosas, Owen-Edwards señala: "Lever Brothers, often praised with pioneering brand identity and marketing, created the first product placement recorded on film. The Swiss businessman and photographer/early film pioneer Francois Henri Lavanchy-Clark (1848-1922) was the representative of Lever Brothers in France and Switzerland. He was an entrepreneur who saw the business potentials of 'product placement' within the moving image from the outset. After entering into a partnership with the Lumiere Brothers he agreed to share his technological inventions if he received the contract to both make and screen Lumiere films in Switzerland".

2 Ya desde finales de siglo, la compañía Coca-Cola promovía su producto con el apoyo de celebridades; la primera de las actrices quizá fuese Hilda Clark y, una de sus primeras intervenciones, un almanaque de 1899 en el que Hilda hace hincapié en las cualidades "deliciosas y refrescantes" de la bebida, a la vez que mantiene que la bebida "alivia el cansancio físico y mental" y "cura la jaqueca" (cfr. Pendergrast 1993: 95).

\section{Los autores}

Juan Salvador Victoria Mas es Profesor Titular de Publicidad y Comunicación Audiovisual. Departamento de Comunicación Audiovisual y Publicidad de la Facultad de Ciencias de la Comunicación de la Universidad de Málaga. Director de ComInteractiva (Congreso Internacional de Comunicación Interactiva). Profesor visitante de la University of Illinois (EEUU), Northwestern University (EEUU), Institute of Higher European Studies (Holanda), Warsaw School of Social Sciences and Humanities (Polonia), Universidad Internacional de Catalunya (España), Universidad Bolivariana de Medellín (Colombia) y Universidad Realística de México. Asesor de empresas de comunicación a nivel internacional (McCann-Ericksson, Audi) y nacional (Grupo Ingenia, Ari Telecom, Dinacom Consulting). Autor de seis libros y medio 
centenar de artículos en relación con la comunicación audiovisual, comercial e interactiva.

Alfonso Méndiz Noguero es Profesor Titular de Teoría e Historia de la Publicidad en el Departamento de Comunicación Audiovisual y Publicidad de la Facultad de Ciencias de la Comunicación de la Universidad de Málaga. Licenciado y Doctor en Ciencias de la Comunicación, ambos con Premio Extraordinario. Diploma en "Production in Motion Pictures \& Television" por la Universidad de California Los Angeles (UCLA). Ha dirigido 12 Tesis Doctorales, cuatro de ellas sobre product placement, y es autor de 14 libros, entre ellos: Modelos de consumidor en la publicidad, Nuevas formas publicitarias, Publicidad y marketing en Internet, Cómo se hicieron las grandes películas (2 vol.). Director del Grupo de Investigación "Nuevas Formas Publicitarias y Nueva Economía (SEJ 396) y Presidente de la Asociación UNESCO para la Promoción de la Ética en los Medios de Comunicación (AUPEMEC).

José Borja Arjona Martín es Profesor del Área de Comunicación en el Centro Universitario Villanueva de Madrid (adscrito a la Universidad Complutense) y Profesor Asociado en la Universidad Internacional de La Rioja. Pertenece al grupo de investigación Nuevas Formas Publicitarias y Nueva Economía (SEJ-396) de la Universidad de Málaga, en el que ha trabajado para diversas publicaciones científicas. 